\title{
A FRAMEWORK FOR INTERPRETING KNOWLEDGE, ATTITUDE AND PRACTICES IN POISONING INCIDENTS OF HOUSEHOLD CHEMICAL PRODUCTS
}

\author{
INDAHAYU AB RAHMAN ${ }^{1}$, MOHAMAD SHAHARUDIN SAMSURIJAN ${ }^{2}$, NUR AZZALIA \\ KAMARUZAMAN $^{1}$, NORHANIZA AMIL ${ }^{3}$ AND MOHD HAFIIDZ JAAFAR ${ }^{1,3 *}$
}

${ }^{1}$ National Poison Centre, ${ }^{2}$ School of Social Sciences, Universiti Sains Malaysia, 11800 Pulau Pinang, Malaysia. ${ }^{3}$ School of School of Industrial Technology, Building G07, Persiaran Sains, Universiti Sains Malaysia 11800 Pulau Pinang, Malaysia.

*Corresponding author: mhafiidz@usm.my

Submitted final draft: 8 August $2020 \quad$ Accepted: 9 August 2020

http://doi.org/10.46754/jssm.2021.07.008

\begin{abstract}
Poisoning by accidental or intentional ingestion, injection or inhalation of household chemical poisons are the most common cases of poisoning reported to the Malaysia National Poison Centre. In Malaysia, there was a notable increase of approximately 94\% of poisoning incidents through ingestion at home. Most poisoning incidents can be extrapolated from a community's demographic factors. The objective of this study is to construct a conceptual framework of knowledge, attitude, and practices (KAP) for household chemical product poisonings. This manuscript is prepared with the focus of improving community health and well-being, as suggested in Goal 3 of the Sustainable Development Goals. In the initial stage of constructing the framework, data sources were gathered from the National Poison Centre (primary data source), and a comprehensive electronic-based search was conducted on the frequent and common keywords to identify scientific reports related to this topic of study. The framework of the developed KAP contains four main sections, which are demographic variables, knowledge, attitude, and practices. At the end of the study, a population's level of awareness of household chemical product poisoning can be measured based on the defined context of the KAPs framework.
\end{abstract}

Keywords: Household chemical, poisoning, knowledge, attitude, practice.

Abbreviations: KAPs (Knowledge, attitudes and practices study), WHO (World Health Organization).

\section{Introduction}

According to the World Health Organisation (WHO) (2000), poisoning incidents have become a significant problem worldwide, particularly in developing countries. In 2020, the Global Burden of Disease Study stated that almost 42,848 poisoning cases were reported to the authorities worldwide (Barratt, 2020). However, not all cases of poisoning were reported to poison centres, and the number of incidents may be higher as most cases of poisoning actually go unreported. Therefore, poisoning data fell short of representing the actual occurrence of poisoning in a country (Bundotich \& Gichuhi, 2015; G/Mariam \& Gelaw, 2016). The issue of poisoning has worsened over time as newer drugs and chemical are gradually developed and introduced.
Household chemical product poisoning by accidental or intentional ingestion, injection or inhalation is the most commonly reported cases of poisoning in medical emergency departments (Kassiri et al., 2012; Adnan et al., 2013; Tangiisuran et al., 2017). Household chemical products, such as cleaning products and toiletries, are commonly used items and can be easily found in all homes all over the world. Products such as bleach, cleaners, disinfectants, and detergents have become essential in maintaining the cleanliness and hygiene of daily human lives. Since the chemical contents of these products are often harmful and poisonous, they can pose serious risks to people's health and environment. Nearly all of the household solution contain approximately $22 \%$ of harmful chemicals, such as formaldehyde and sodium 
chloride (EWG's Guide to Healthy Cleaning, n.d), which could promote a range of adverse side effects, such as nausea, burning sensation, coughs, and sore throat. Therefore, these household chemical products must be used according to their instructions to ensure the safety of their users and surroundings.

Unfortunately, these household cleaning products have become one of the most common reagents that have caused an increase in poisoning incidents globally, whether unintentionally or intentionally. Adinew et al. (2017) reported that $25 \%$ to $35 \%$ of 543 poisoning cases involved patients poisoned with bleaching reagents. Meanwhile, Mckenzie et al. (2010) stated that household cleaning products were responsible for many unintentional poisoning cases among children and consistently remained among the top causative agents for paediatric poisoning exposure. The causes of household chemical product poisoning are multi-factorial, and thus must be analysed further to understand them and so that strategies for better prevention can be devised.

Poisoning incidents caused by household chemical products occur due to the exceeding level of toxicity and the duration of exposure to the products (Cleveland Clinic, 2018). The two categories of household chemical product poisoning are unintentional and intentional. Unintentional poisoning is defined as the exposure by any route where there is no intention to cause harm (Tangiisuran et al., 2018), and children are prone to this kind of poisoning. Deaths from unintentional poisoning commonly occur in low- and middle-income groups (Gorea, 2009; Adnan et al., 2013; Tangiisuran et al., 2017). According to data from the WHO (2012), an estimated 193,460 people died worldwide from unintentional poisoning in 2012. These incidents had lowered the quality of life globally in the aspect of health. Carelessness in handling household chemical products and the lack of concern by family members in regards to the urgency of this issue are the most common factors that contribute to many unintentional cases of poisoning (Dayasiri et al., 2018; Peshin \& Gupta 2018).
Intentional poisoning can be classified as suicidal cases and involves the deaths of nearly a million people each year (WHO, 2019). This poisoning incident category can be defined as exposure by any route where there is an intention to cause harm (Tangiisuran et al., 2018). It is estimated by the WHO (2008) that deliberate ingestion of household chemical poisons caused 370,000 deaths per year. Moreover, of the 2-3 million of poisoning cases annually, about 2 million was estimated to be annual intentional poisonings that resulted in about 200,000 suicides (Moazzam et al., 2009; Banerjee et al., 2012; G/Mariam \& Gelaw, 2016). The high occurrence rates may be attributed to ethnicity, cultural and geographical factors (Azizpour et al., 2016). Generally, most intentional poisoning cases involve people in the age group of 15-19 years old (Adnan et al., 2013). Most previous studies reported that males were significantly involved in intentional poisoning based on the high number of poisoning exposure calls recorded (Jesslin et al., 2010; Rajapakse et al., 2014).

In a developing country like Malaysia, approximately $96 \%$ of poisoning exposure cases (household products, pharmaceutical products, and pesticides were the common agents) were reported to have occurred at home (Tangiisuran et al., 2017). The unintentional and intentional poisoning cases from the National Poison Centre (NPC) database as referred to in Figure 1 can be further analysed with the extrapolation of demographic factors of the human population to prevent or lower incidents of poisoning (Marks \& Hoving, 2016). In this paper, a framework was constructed to identify the epidemiology of poisoning, as well as the geographical and individual factors (knowledge, attitude, and practices) in a defined population.

According to the NPC, more than 50,000 poisoning cases were referred for inquiries from 1995 to 2017 (Figure 1), while 53\% of the poisoning incidents from 2006 to 2016 were caused by household chemical products (Figure 2). Since then, this centre has observed an annual increase in the number of poisoning cases referred. 


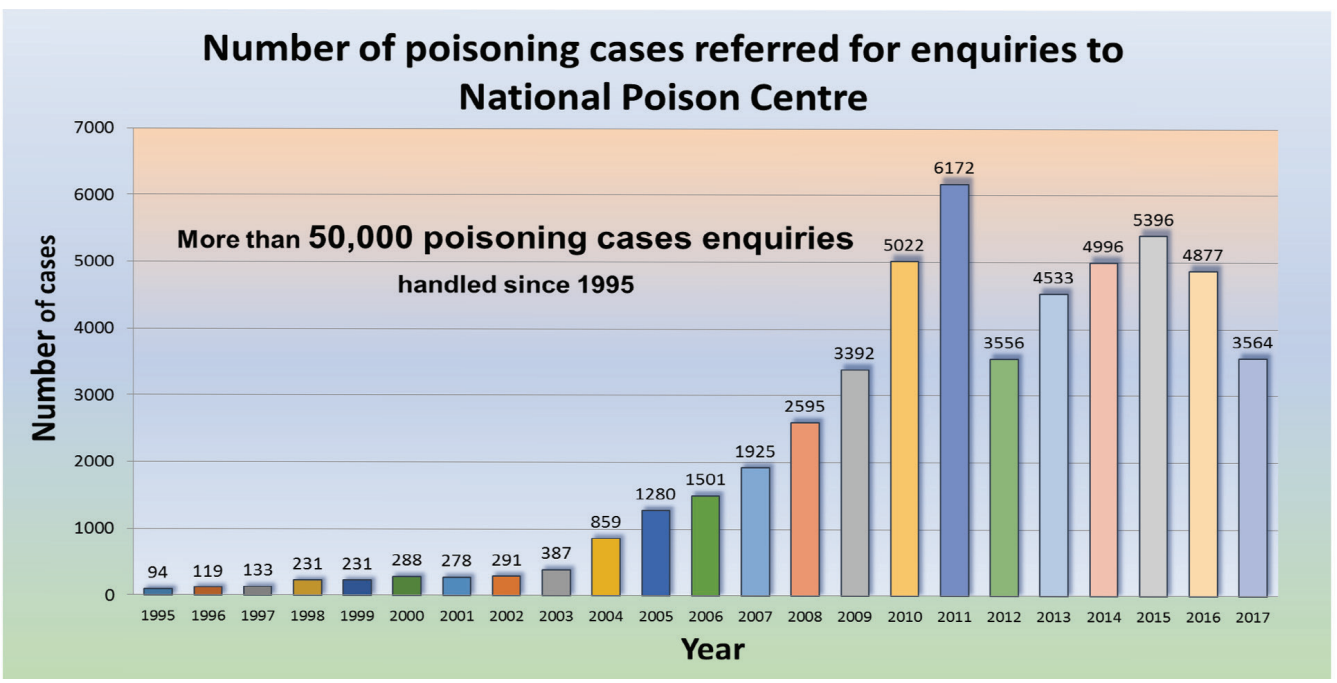

Figure 1: The number of poisoning cases reported to the National Poison Centre (NPC) from 1995 to 2017.

(Source: National Poison Centre)

\section{Number of household product poisoning cases referred to NPC for enquiries in 2006-2016}

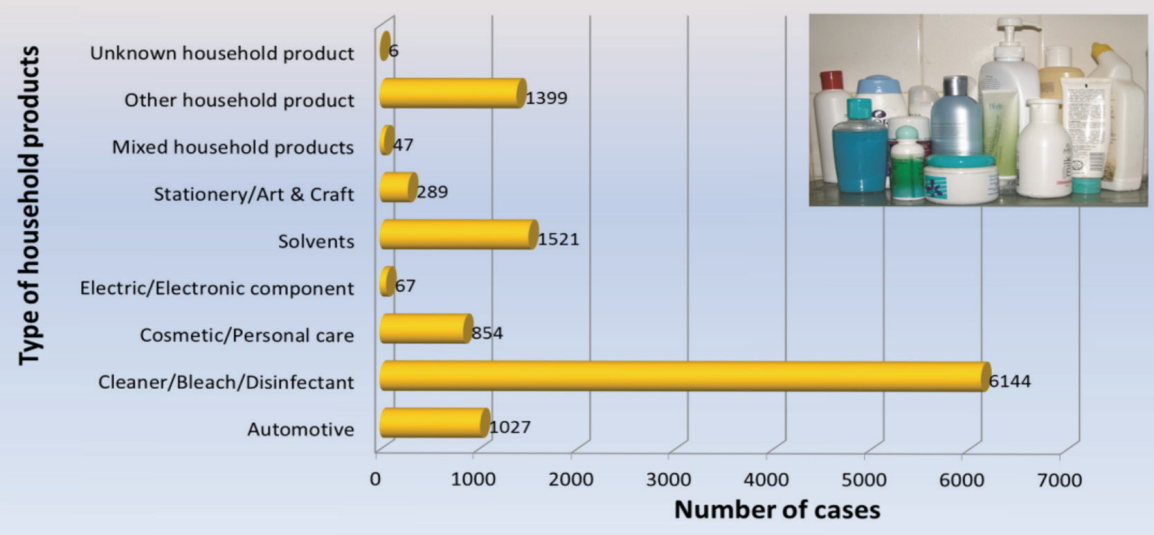

Figure 2: The number of poisoning cases reported to the National Poison Centre from 2006-2016, grouped by the type of poisoning reagent. (Source: National Poison Centre)

\section{Methodology}

In constructing the framework of the knowledge, attitude, and practice study (KAP study) on household chemical poisoning, the NPC database became the primary data source, and a retrospective review of the database from 1995 to 2017 was conducted. This centre had also noted an annual increase in the number of poisoning cases referred. Household cleaning products were recorded as the household chemical reagent that caused the highest number of poisoning incidents, followed by solvents (thinner and turpentine) and other household products.

Following the initial study of the NPC database, a comprehensive electronic-based 
search was performed on the frequent and common keywords to identify scientific reports related to this topic of study. Selected publications from the past 12 years were reviewed. Other relevant articles and older publications were also studied, with no language restriction for any publication. The search terms included the words "household chemical products", "children", "poisoning", "unintentional and intentional", "KAP study in poisoning" and the most common one was "household cleaning products". The journals that were referred to in this study were ranked using the Scimago Journal and Country Rank. Thus, journals with rankings of Q1- Q4 were reviewed in this study.

\section{Results and Discussion}

\section{The KAP framework for household chemical product poisoning}

Figure 3 depicts the conceptual framework of the KAP derived from all inputs and information gathered during the data searching and sourcing process. The framework was aimed to identify indicators that can help researchers improve the development and implementation of poisoning awareness. The developed KAP's framework contains four main sections, which are demographic variables, knowledge, attitude, and practices. Demographic variables and the KAPs can be indicators of the risk of poisoning (Presgrave et al., 2008). The variable patterns of poisoning cases mostly depend on the demographic variables of the individual itself

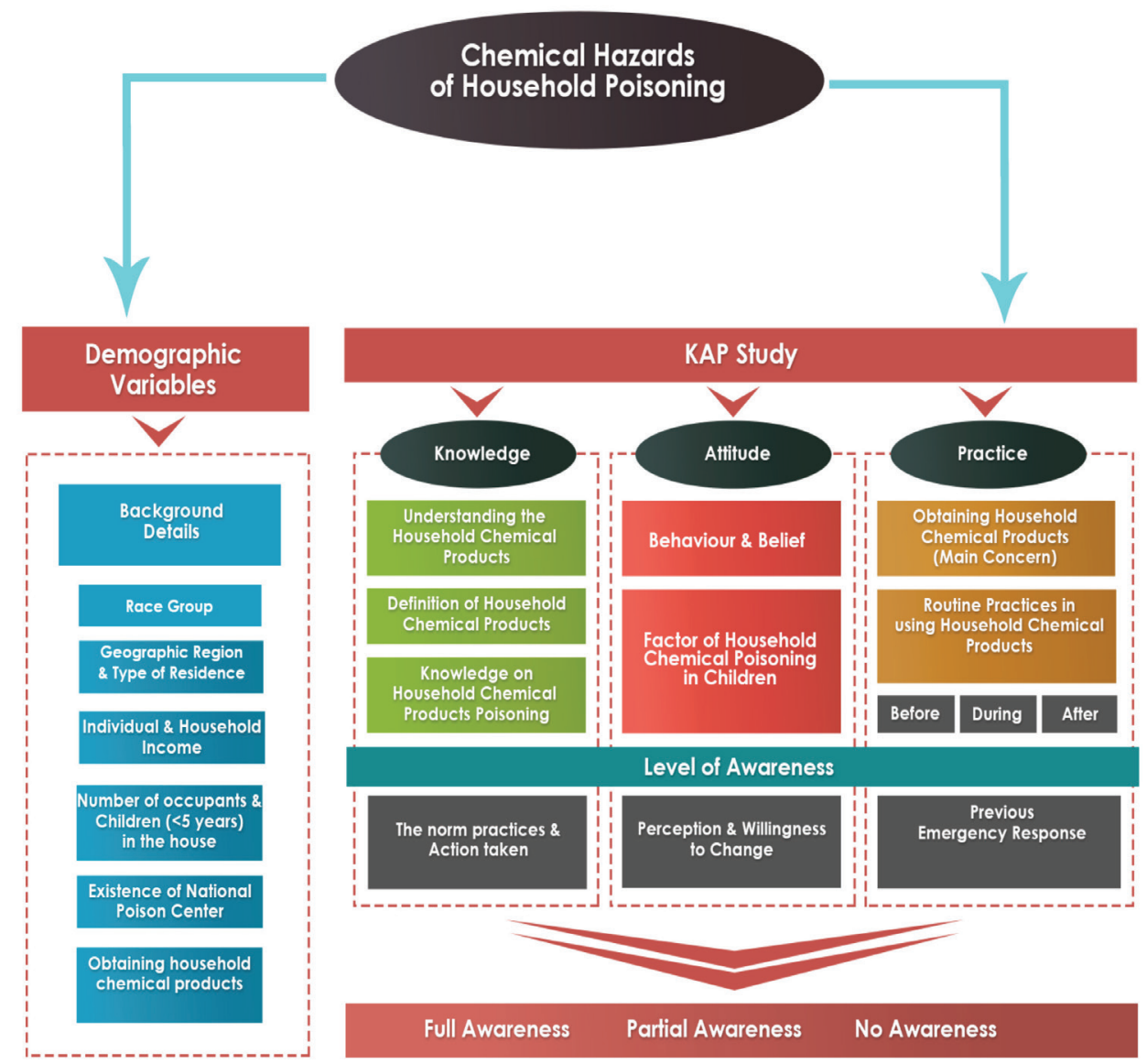

Figure 3: The conceptual framework of the knowledge, attitude, and practice (KAP) on household chemical products poisoning. 
(Peshin \& Gupta, 2018). In Egypt, many children in rural areas are affected by home injuries, especially poisonings, and the associated factors of these incidents are their parents' knowledge, attitude, and practices (Eldosoky, 2012).

At the end of the framework, the level of awareness of the population is measured within the KAP's contexts. The final results categorise the population into three groups, which are those with full awareness, partial awareness, and no awareness. Each context has its indicators or inputs to evaluate the particular population. Awareness of issues of household chemical product poisoning among the population has not been well described. Thus, this study could address problems of actual local relevance.

\section{Demographic Variables}

Demographic variables are an essential component of this KAPs framework (Figure 3, the Demographic Variables part) as the epidemiology data provide information on the respondents that may help this study determine the factors influencing household chemical poisoning (Manzar et al., 2010; World Health Organisation (WHO), 2010; Jarrah et al., 2018; Sena et al., 2018). Previous research had proven that a variety of social and demographic variables (such as geographic location, age of respondents, gender, education background, household size, residential characteristics, ethnic group, income and marital status) has an influence on unintentional poisoning cases (Centers for Disease Control and Prevention (CDC), 2006; Franklin \& Rodgers, 2008; Gheshlaghi et al., 2013).

The epidemiology data of an individual in a defined population that are correlated with the topic of study are included in the framework. The background details as shown in Figure 3 that are needed in this study are gender (male or female), age, education level, and marital status. Some studies have shown that these details needed to be taken into consideration as these variables could have an influence on the occurrence of poisoning (Manzar et al., 2010). For example, it was found that most individuals with a higher level of education were prone to have children being involved in household poisoning cases (Manzar et al., 2010). This is because the children were neglected as a result of busy and workaholic parents who strived for career advancement (Manzar et la., 2010). In 2016, the average household size in Malaysia is 4.1 (Department of Statistics Malaysia, 2017). It is explained that most households in Malaysia have at least four people, who can be classified as the core family members. Sackitey (2018) found that the frequency of home accidents was lower in children who live in a core or nuclear family compared with those living with an extended family. In addition, Kielb et al. (2015) said that indoor air quality could be affected if the number of occupants in a house was overloaded.

Manzar et al. (2010) had suggested that a large scale of variables, like ethnicity and geographic differences, for household poisoning cases were needed in future studies as they believed that these factors could have an impact on different cultures and practices. Lam (2003) had suggested that people in rural areas could have a higher chance of exposure to poisons due to the prevalence of agricultural activities in these areas. Poisoning incident factors may vary according to region, societal (ethnic) tradition and customs, level of education, and season (Sivri \& Ozpulat, 2015). It was also reported that people with a functional economic status would have a higher level of knowledge and awareness of household chemical poisoning (Sivri \& Ozpulat, 2015). Therefore, this study had included the variable of household income based on the group classification as defined by the Department of Statistics Malaysia (DSM, 2017). There are four groups of household income, which are poverty (measured by the Poverty Line Income, PLI), B40 ( $\geq$ RM 2,848), M40 ( $\geq$ RM 6502), and T20 ( $\geq$ RM 16,088). This study also considers the number of children in a household aged between 0 and 5 years old as most of the reported poisoning cases involved this age group (Rosenberg et al., 2011; Akturk \& Erci, 2016; Peshin \& Gupta, 2018). Children below the age of 5 years old were a major concern in this study as they are particularly 
active and have an exploratory nature with a strong impulse to put things into their mouths. Moreover, living with parents who are likely to be inattentive and neglectful exacerbated their vulnerable situation (Hunt, 2013).

\section{Knowledge}

In the KAP surveys, knowledge is one of the main aspects that will define the extent of community awareness of public health concepts. Every person has a different level of knowledge on the chemical hazards of household poisoning. Availability of such information would allow poison management control centres to plan preventive interventions to educate the community efficiently. Goktas et al. (2014) evaluated respondents' knowledge on poisoning cases involving household chemical products. It was found that the respondents' knowledge was defined by their health education background and level of awareness of this crucial issue. The WHO (2005) believed that an individual's high level of knowledge is one of the preventive measures and most significant component that influences responses to poisoning cases. It is crucial because household chemical product poisonings can cause mortality and morbidity in humans.

The context of knowledge in this study is to assess the extent to which individuals from a household are aware of public health concepts regarding the use of household chemical products (as shown in Figure 3, part: knowledge). Focus group questions further contextualised a targeted community's knowledge on poisoning and understanding of household chemical product management. A study by Bogar et al. (2017) showed that the majority of people had minimal knowledge of prevention strategies and were misinformed about poisoning (Bogar et al., 2017). This study will not be limited to knowledge of biochemical facts, but will focus on general knowledge that could lead to the measurement of the level of public awareness and literacy, such as knowledge on the type of household chemical products and poisoning risk assessments. Questions on respondents' understanding were developed for this study, such as "Can they differentiate well the hazardous household chemical products in their house?" and "Are they aware of and do they know the factors that may lead to poisoning?"

Education background and understanding of household chemical poisoning (potential risks of accidental poisoning in a home environment) of guardians (parents, grandparents and caregivers) or those entrusted with the care of children and other individuals are essential in responding to poisoning incidents (Peshin \& Gupta, 2018). Thus, this study included education background as a variable because it could have an influence on the level of an individual's knowledge of the risks of household chemical poisoning. According to the National Safety Council (2019), the public should make an informed decision about the type of household products they buy and bring into their homes. They also need to understand very well the terms and definition found on the products to avoid any poisoning incident. Parents' knowledge of poisoning is essential in injury care for children as many adverse consequences of poisoning incidents can be averted if parents know how to respond to them (Ibrahim, 1991; Eldosoky, 2012).

Understanding household chemical products will reduce the possibility of poisoning incidents in selected populations. This study identified the main risk factors of unintentional and intentional poisoning incidents in the investigated population. A further focus was given to finding the possible causes of household chemical poisoning incidents. Many studies were conducted on the causes of poisoning in different parts of the world. Most poisoning cases occurred because of ingestion of household chemicals (Mansori et al., 2016). The increasing number of poisoning cases is due to the introduction of new and various household chemical products, the increasing number of new consumer products and greater accessibility and exposure of children to these products (Balai-Mood, 2004; Mansori et al., 2016). 
In this context of knowledge, the norm practices and responses to poisoning incidents are also considered in the measurement of level of awareness. Most people live with dangerous poisons in their household. This living norm increases the risks of poisoning if household chemical products are not well managed (Rosenberg et al., 2011; National Safety Council, 2019). Referring to Figure 3, the last part of knowledge, familiarity versus awareness, means that many people have norm practices in their life that are based on familiarity without any concern for awareness. Some people are unable to recognise that their norm practices could bring physical and health hazards to their household. Most parents and caregivers have bad habits when managing household chemical products and this puts their children's lives at risk. An example of a bad habit is keeping hazardous household chemical products in the wrong places or unlatched storage spaces in the house (Gutierrez et al., 2011).

\section{Attitude}

Attitude is difficult to define, and even within the field of psychology, there is little agreement as to what is understood by it (Mbeng et al., 2009). In this study, however, the context of attitude was focused on determining beliefs, behaviours, and attitudinal factors in relation to household chemical poisoning. Attitude can be defined as a complex mental state involving belief, feelings, and values, which all facilitate a predilection to behave or act in a particular way (Sharif \& Al-Maliki, 2010; Akabanda et al. 2017). Attitude may be attributed to the characterisation of an individual's feelings and inclinations with regards to the use of household chemical products (Muleme et al., 2017). The characterisation can be defined as negative (bad) or positive (good) in relation to the scientifically documented risks of household chemical product poisoning. The translation of correct knowledge into practices can be used to reduce poisoning cases through a change of belief (attitude) concerning household chemical hazards (Sharif \& Al-Malki, 2010). Figure 3 (the
Attitude part) illustrates the context of attitude in this framework.

Behaviour and belief are related to an individual's attitude. One part of the crosssectional study by Rosenberg et al. (2011) rated people's beliefs on the poisonous levels of household chemical products and from that, the attitudinal scale could be measured. The rate of beliefs on hazardous household items could show how much people recognise and are concerned about the potential of household chemical products in causing harm and severe poisoning to their family, especially children. It was believed that household or laundry cleaning products were among the products that lead to poisoning incidents (Presgrave et al., 2008; Rosenberg et al., 2011; Tangiisuran et al., 2018).

Every year, more than 1 million children below 5 years old experience potentially toxic ingestions (Watson et al., 2004; Lai et al., 2006; Franklin \& Rodgers, 2008). Poisoning may occur in common locations, like the home, often in the living/dining room, kitchen, or the bedroom, as these are the locations where children (potential victims) spend most of their time (Bertinelli et al., 2006; McKenzie et al., 2010). Children in this age range are the group that are most vulnerable to household chemical poisoning (Awang et al., 2003; Lam, 2003; Gutierrez et al., 2011; Tangiisuran et al., 2018). They have a high prevalence of acute poisoning as they are likely to taste or swallow harmful substances (Haghighat \& Moatamedi, 2012; Adnan et al., 2013) due to their behaviour; curious, exploratory in nature, and prone to putting things in their mouths (typical of early childhood development) (Lekei et al., 2017; Peshin \& Gupta, 2018). Therefore, strict supervision by adults is mandatory, and the reflection of attitude and perception (belief) of parents may affect the course of preventive action taken (Rosenberg et al., 2011). Moreover, parents must teach the right attitude towards effective and safe management and practices of household chemical products at home.

In the attitude context, the level of awareness could be measured from a population's perceptions and willingness to 
change. A study by Rosenberg et al. (2011) indicated that the majority of their respondents did not intend to change themselves to reduce the risk of poisoning. It was also reported that individuals who had completed university education might have more awareness and motivation to prevent the occurrence of poisoning (Eldosoky, 2012). Therefore, it is important to perform epidemiology surveillance to assess the magnitude of poisoning issues and identify significant risk factors to instil positive attitude with the right beliefs and perceptions among the people (Presgrave et al., 2008). To ensure an effective reduction of poisoning risks, initiating changes to the fixed environment must be supported by enforcement of regulation, adequate education for industries and the community, as well as clear labelling of the products (Nixon et al., 2004). In short, bad or good individual attitude can be affected by their surroundings, like the presence of economic, marital, or sibling-related problems, and family social support (Dayasiri et al., 2017).

\section{Practices}

Practices in this study as referred to in Figure 3 (Practice part) indicates any action related to household chemical products, right from purchasing to usage, management, and storage. In short, practices can be defined as the real action taken based on knowledge and attitude (Agbedia, 2013). The right and suitable knowledge gained from dependable sources could change people's attitude and lead to proper practices that decrease the risk of poisoning (Sharif \& Al-Malki, 2010). Ideally, most people are more inclined to practise the right procedures if they are backed by scientific or evidence-based information (Brownson et al., 2009). In developing proper practices to overcome poisoning cases, household chemical product management needs to be implemented appropriately (Lekei et al., 2017).

There are a variety of household chemical products in the market. It is expected that most people prefer products that are of the highest quality (Muleme et al., 2018). Places where the study population buy their products play a significant role in their purchase decisions. According to Maciejewski (2016), people were more likely to go to supermarkets or hypermarkets to purchase cleansing products as large-scale retailers sell various brands and types of basic household and cleaning products (Hassan et al., 2013).

Many people have shown poor knowledge of home poisoning and a lack of knowledge on the proper practices in the use of household chemical products, which led to poor practices of poisoning prevention strategies (Gutierrez et al., 2011). As a result, vulnerable and innocent children end up becoming the ultimate victims. A study suggested that poisoning prevention strategies should be about creating awareness, as well as listing the essential dos and dont's for household chemical usage (Peshin \& Gupta, 2018). The strategies should also be evident in dictating safe routine practices before, during, and after the usage of household chemical products. Significant contributing factors need to be addressed through prevention programmes and measures to reduce the risks of poisoning. Common practices, like improper storage and careless handling of household chemical products, pose a higher risk of poisoning incidents at home (Gorea, 2009). For example, some people have a habit of storing their household cleaning products under the sink in the kitchen or on the floor in the backyard, which are within the reach of children.

As observed in Figure 3, for the practice part, a census was created to measure the targeted population's experience of household chemical poisoning in a particular community. Data from this census can be analysed to conclude the actions taken when poisoning occurs (Rosenberg et al., 2011). For example, the appropriate use of immediate first aid is vital during a poisoning emergency as this could save a person's life or lower the severity of poisoning. This particular action could be evaluated in this study to determine a respondent's action in response to poisoning incidents at home. On another note, it has been reported that 
$41 \%$ of callers from Puerto Rico in 2008 who sought advice on home poisoning incidents had completed first-aid courses and had filled in reports to their respective Poison Information Centres (Gutierrez et al., 2011). This further highlights the importance of home poisoning prevention knowledge and the role of poison control centres as effective strategies to guide people to respond to emergency home poisoning incidents with suitable and efficient practices that could save human lives.

\section{Conclusions}

Household chemical products are considered vital in our daily lives, and products of various types can be found in almost all homes. However, easy accessibility and careless storage, coupled with trivial parental supervision, are becoming the core causes of accidental children poisoning incidents. The differences observed in the poisoning reagents responsible for both accidental and intentional poisoning incidents in different countries and different communities depend on social status and prevalent cultural practices. As noted above, knowledge alone may not be adequate to prevent poisoning incidents as positive attitudes and correct practices are also needed for more effective interventions. Children are particularly affected when it comes to poisoning due to differences in the aspects of physiology, behaviour, and biological defences, thus making them vulnerable to unintentional poisoning by ingestion. Hence, poisoning incidents can only be reduced by broad-based preventive strategies, including enforcement of safe manufacturing practices (like child locked cap on a product) and public education programmes (through campaigns and media). Furthermore, poison prevention measures must essentially shift from self-education of adults to advocating the correct knowledge, attitude and practices to children.

\section{Acknowledgments}

This research study was supported financially by the Penang Government and MRUN -
Translational Research Grant with the reference numbers of PRO-S-1012-I060 and 304. PTEKIND.656207. The authors would like to extend gratitude to colleagues who provided insights and expertise that majorly assisted the research study.

\section{References}

Adinew, G. M., Woredekal, A. T., DeVos, E. L., Birru, E. M., \& Abdulwahib, M. B. (2017). Poisoning cases and their management in Emergency Centres of Government Hospitals in Northwest Ethiopia. African Journal of Emergency Medicine, 7(2), 7478. doi:10.1016/j.afjem.2017.04.005

Adnan, L. M., Kamaldin, J., Mohamad, N., Salatore, S. A., Suhaimi, R., Zainuddin, N., \& Mohamad Shariff, M. H. (2013). The risk of accidental chemical poisoning cases among children ( $\leq 12$ years old) admitted to Hospital University Sains Malaysia: 5 years review. Journal of Clinical Toxicology, 3(5), 3-7. doi:10.4172/2161-0495.1000177

Agbedia, C. (2013). Knowledge, attitude and practice (KAP) of sandwich under graduate students of Delta State University, Abraka, Nigeriaon condom use in the prevention of sexually transmitted infections (STls) and unwanted pregnancy. IOSR Journal of Humanities and Social Science, 7(4), 7378. doi: 10.9790/0837-0747378

Akabanda, F., Hlortsi, E. H., \& OwusuKwarteng, J. (2017). Food safety knowledge, attitudes and practices of institutional food-handlers in Ghana. $B M C$ Public Health, 17(1). doi:10.1186/s12889016-3986-9

Akturk, U., \& Erci, B. (2016). Determination of knowledge, attitudes and behaviors regarding factors causing home accidents and prevention in mothers with a child aged 0-5 years. Journal of Education and Practice, 7(18), 142-153. doi:10.1002/14651858. CD009882.pub2 
Awang, R., Abd Rahman, A. F., A Wan Abdullah, W. Z., Lajis, R., \& Abdul Majid, M. I. (2003). Trends in inquiries on poisoning: A five-year report from the National Poison Centre, Malaysia. Medical Journal of Malaysia, 58(3), 375-379. Retrieved May 14, 2019.

Azizpour, Y., Asadollahi, K., Savehmiri, K., Kaikhavani, S., \& Abangah, G. (2016). Epidemiological survey of intentional poisoning suicide during 1993-2013 in Ilam Province, Iran. BMC Public Health, 16(902), 1-12. doi: 10.1186/ s12889-016-3585-9

Balai-Mood M. (2004). Pattern of acute poisonings in Mashhad, Iran 1993-2000. Clinical Toxicology, 42(7), 965-75.

Banerjee, I., Tripathi, S. K., \& Roy A. S. (2012). Clinico-epidemiological characteristics of patients presenting with organophosphorus poisoning. North American Journal of Medical Sciences, 4(3), 147-150.

Barratt, I. (2020). Global deaths due to various causes and COVID-19. Retrieved July 13, 2020.

Bertinelli, A., Hamill, J., Mahadevan, M., \& Miles, F. (2006). Serious injuries from dishwasher powder ingestions in small children. Journal of Paediatrics and Child Health, 42(3), 129-133. doi:doi:10.1111/ j.1440-1754.2006.00811.x

Bilgen Sivri, B., \& Ozpulat, F. (2015). Mothers' knowledge levels related to poisoning. Turkish Journal of Emergency Medicine, 15(1), 13-22. doi:10.5505/1304. 7361.2014 .25582

Bogar, S., Szabo, A., Woodruff, S., \& Johnson, S. (2017). Urban youth knowledge and attitudes regarding lead poisoning. Journal of Community Health, 42, 1255-1266. doi: 10.1007/s10900-017-0378-8

Brownson, R. C., Chriqui, J. F., \& Stamatakis, K. A. (2009). Understanding evidencebased Public Health Policy. American
Journal of Public Health, 99(9), 15761583. doi:10.2105/ajph.2008.156224

Bundotich, J. K., \& Gichuhi, M. M. (2015). Acute poisoning in the Rift Valley Provincial General Hospital, Nakuru, Kenya: January to June 2012 JK. South African Family Practice, 57(3), 214-218. doi:10.1080/207 86190.2014 .975448

Centers for Disease Control and Prevention (CDC). (2006). Nonfatal, unintentional medication exposures among young children: United States, 2001-2003. MMWR Morb Mortal Wkly Rep, 55(1), 1-5.

Cleveland Clinic. (2018, May 24). Household Chemical Products and Their Health Risk. Retrieved December 6, 2018, from https://my.clevelandclinic.org/health/ articles/11397-household-chemicalproducts-and-their-health-risk

Dayasiri, M. B. K. C., Jayamanne, S. F., \& Jayasinghe, C. Y. (2018). Patterns and outcome of acute poisoning among children in rural Sri Lanka. BMC Pediatrics, 18(274), 1-8. doi: 10.1186/s12887-018-1246-0

EWG's Guide to Healthy Cleaning. (n.d.). Household cleaner ratings and ingredients. Retrieved July 22, 2020.

Eldosoky, R. S. H. (2012). Home-related injuries among children: Knowledge, attitudes and practice about first aid among rural mothers. Eastern Mediterranean Health Journal, 18(10), 1021-1027. doi:10.26719/2012.18.10.1021

Franklin, R. L., \& Rodgers, G. B. (2008). Unintentional child poisonings treated in United States Hospital Emergency Departments: National Estimates of incident cases, population-based poisoning rates and product involvement. Pediatrics, 122(6), 1244-1251. doi:10.1542/peds.20073551

G/Mariam, E. T., \& Gelaw, B. K. (2016). Global epidemiology of acute poisoning with an emphasis to Ethiopia: Systematic review. International Journal of Pharma Sciences 
and Scientific Research, 2(4), 120-130. doi:10.25141/2471-6782-2016-4.0161

Gheshlaghi, F., Piri-Ardakani, M., Yaraghi, M., Shafiei, F., \& Behjati, M. (2013). Acute poisoning in children; A population study in Isfahan, Iran, 2008-2010. Iranian Journal of Pediatrics, 23(2), 189-193.

Goktas, S., Yildirim, G., Kose, S., Yildirim, S., Ozhan, F., \& Senturan, L. (2014). First aid knowledge of University students in poisoning cases. Turkish Journal of Emergency Medicine, 14(4), 153-159. doi: 10.5505/1304.7361.2014.15428

Gorea, R. (2009). Prevention and current scenario of unintentional poisoning. Journal of Punjab Academy of Forensic Medicine \& Toxicology, 9, 54-57.

Gutierrez, J., Negrón, J., \& García-Fragoso, L. (2011). Parental practices for prevention of home poisoning in children 1-6 years of age. Journal of Community Health, 36(5), 845-848. doi:10.1007/s10900-011-9384-4

Hasliza, H., Sade, A. B., \& Rahman, M. S. (2013). Malaysian hypermarket retailing development and expansion. International Journal of Retail and Distribution Management, 41(8), 584-595. doi: 10.1108/ IJRDM-09-2012-0085

Hunt, J. C. (2013). Associations between different parenting styles and child behaviour (Doctoral dissertation, Philadelphia College of Osteopathic Medicine) (pp. 1-66). Pennsylvania: PCOM Psychology Dissertations.

Ibrahim A. (1991). Assessment of knowledge, attitude and practice of mothers attending Cairo University Hospital toward home accidents among preschool children (MSc thesis). Cairo, Egypt, Higher Institute of Nursing, University of Cairo.

Jarrah, S., Judeh, M., \& AbuRuz, M. E. (2018). Evaluation of public awareness, knowledge and attitudes towards basic life support: A cross-sectional study. BMC Emergency
Medicine, 18(37), 1-7. doi: 10.1186/ s12873-018-0190-5

Jesslin, J., Adepu, R., \& Churi, S. (2010). Assessment of prevalence and mortality incidences due to poisoning in a South Indian Tertiary Care Teaching Hospital. Indian Journal of Pharmaceutical Sciences, 72(5), 587-591.

Kassiri, H., Haddad, M. H., Ghasemi, F., Rezaei, M., \& Ghanavati, F. (2012). An epidemiologic and demographic survey of poisoning in Southwest of Iran. MiddleEast Journal of Scientific Research, 12(7), 990-996. doi:10.5829/idosi. mejsr.2012.12.7.7155

Kielb, C., Lin, S., Muscatiello, N., Hord, W., Rogers-Harrington, J., \& Healy, J. (2015). Building-related health symptoms and classroom indoor air quality: A survey of school teachers in New York State. Indoor Air, 25(4), 371-380. doi:10.1111/ ina.12154

Lai, M. W., Klein-Schwartz, W., Rodgers, G. C., Abrams, J. Y., Haber A. B, D. A., Bronstein, A. C., \& Wruk R. N, K. M. (2006). 2005 Annual Report of the American Association of Poison Control Centers' National Poisoning and Exposure Database. Clinical Toxicology, 44(6-7), 803-932. doi: $10.1080 / 15563650600907165$

Lam, L. T. (2003). Childhood and adolescence poisoning in NSW, Australia: An analysis of age, sex, geographic, and poison types. Injury Prevention, 9(4), 338-342. doi:10.1136/ip.9.4.338

Launiala, A. (2009). How much can a KAP survey tell us about people's knowledge, attitudes and practices? Some observations from medical anthropology research on malaria in pregnancy in Malawi. Anthropology Matters, 11(1), 1-19.

Lekei, E., Ngowi, A. V., \& London, L. (2017). Acute pesticide poisoning in children: Hospital review in selected 
hospitals of Tanzania. Journal of Toxicology, 2017, 1-8. doi:10.1155/2017/4208405

Maciejewski, G. (2016). Purchasing places of consumer goods in selected European countries. Oeconomia, 15(2), 87-101.

Mahidin, M. U. (2017). Press Release Report of Household Income and Basic Amenities Survey 2016(pp. 1-7) (Malaysia, Department of Statistics Malaysia, Jabatan Perangkaan Malaysia). Kuala Lumpur: Public Relation Officer.

Manzar, N., Ali Saad, S. M., Manzar, B., \& Fatima, S. S. (2010). The study of etiological and demographic characteristics of acute household accidental poisoning in children-A consecutive case series study from Pakistan. BMC Pediatrics, 10, 28. doi:10.1186/1471-2431-10-28

Mansori, K., Soori, H., Farnaghi, F., Khodakarim, S., Mansouri Hanis, S., \& Khodadost, M. (2016). A case-control study on risk factors for unintentional childhood poisoning. Medical Journal of the Islamic Republic of Iran (MJIRI), 30(1), 355-362. Retrieved August 1, 2019.

Marks, C. J., \& Hoving, D. J. (2016). A 3-year survey of acute poisoning exposures in infants reported in telephone calls made to the Tygerberg Poison Information Centre, South Africa. South Africa Journal Child Health, 10(1), 43-46. doi:10.7196/ SAJCH.2016.v10i1.1045

Mbeng, L., Probert, J., Phillips, P., \& Fairweather, R. (2009). Assessing public attitudes and behaviour to household waste management in Cameroon to drive strategy development: A Q Methodological Approach. Sustainability, 1(3), 556572. doi:10.3390/su1030556

McKenzie, L. B., Ahir, N., Stolz, U., \& Nelson, N. G. (2010). Household cleaning productrelated injuries treated in US Emergency Departments in 1990-2006. Pediatrics, 126(3), 509-516. doi:10.1542/peds.20093392
Meyer, S., Eddleston, M., Bailey, B., Desel, H., Gottschling, S., \& Gortner, L. (2007). Unintentional household poisoning in children. Klinische Padiatrie, 219(5), 254270. doi:10.1055/s-2007-972567

Moazzam M., Al-Saigul A. M., Naguib M., \& Alfi M.A. (2009). Pattern of acute poisoning in Al-Qassim region: A surveillance report from Saudi Arabia, 1999-2003. Eastern Mediterrian Health Journal, 15(4), 1005 1010.

Muleme, J., Kankya, C., Ssempebwa, J. C., Mazeri, S., \& Muwonge, A. (2017). A framework for integrating qualitative and quantitative data in knowledge, attitude, and practice studies: A case study of pesticide usage in Eastern Uganda. Frontiers in Public Health, 5(318), 1-15. doi:10.3389/ fpubh.2017.00318

National Safety Council. (n.d.). Poisons: What Parents Need to Know. Retrieved June 10, 2019, from https://www.nsc.org/ home-safety/safety-topics/other-poisons/ household-products

Nixon, J. (2004). Community based programs to prevent poisoning in children $0-15$ years. Injury Prevention, 10(1), 43-46. doi:10.1136/ip.2003.003822

Peshin, S. S., \& Gupta, Y. (2018). Poisoning due to household products: A ten years Retrospective Analysis of Telephone Calls to the National Poisons Information Centre, All India Institute of Medical Sciences, New Delhi, India. Journal of Forensic and Legal Medicine, 58, 205-211. doi:10.1016/j. jflm.2018.07.005

Presgrave, R. D., Camacho, L. B., \& Villas Boas, M. S. (2008). A profile of unintentional poisoning caused by household cleaning products, disinfectants and pesticides. Cadernos De Saude Publica, 24(12), 2901-2908. doi:10.1590/S0102311X2008001200019

Rajapakse, T., Griffiths, K. M., Christensen, H., \& Cotton, S. (2014). A comparison 
of non-fatal self-poisoning among males and females, in Sri Lanka. BMC Psychiatry, 14(221), 1-13. doi: 10.1186/ s12888-014-0221-z

Rajasuriar, R., Awang, R., Hashim, S., \& Rahmat, H. (2007). Profile of poisoning admissions in Malaysia. Human \& Experimental Toxicology, 26, 73-81. doi: $10.1177 / 0960327107071857$

Rosenberg, M., Wood, L., Leeds, M., \& Wicks, S. (2011). "But they can't reach that high ...". Parental perceptions and knowledge relating to childhood poisoning. Health Promotion Journal of Australia, 22(3), 217223. doi:10.1071/HE11217

Sackitey, G. L. (2018). Knowledge, attitude and perception on prevention of home accidents among mothers who came to the Pediatrics Department of the Korle-Bu Teaching Hospital. Journal of Health Education Research \& Development, 6(1), 1-10. doi:10.4172/2380-5439.1000242

Sena, B., Zaki, S. A., Yakub, F., Yusoff, N. M., \& Ridwan, M. K. (2018). Conceptual framework of modelling for Malaysian Household Electrical Energy Consumption using Artificial Neural Network based on Techno-Socio Economic Approach Boni. International Journal of Electrical and Computer Engineering (IJECE), 8(3), 1844-1853. doi:10.11591/ijece.v8i3. pp1844-1853

Sharif, L., \& Al-Malki, T. (2010). Knowledge, attitude and practice of Taif University students on food poisoning. Food Control, 21(1), 55-60. doi:10.1016/j. foodcont.2009.03.015

Tangiisuran, B., Jiva, M., Ariff, A. M., Abdul Rani, N., Misnan, A., Rashid, S. M., \& Dawson, A. H. (2018). Evaluation of types of poisoning exposure calls managed by the Malaysia National Poison Centre (20062015): A retrospective review. BMJ Open, 8, 1-7. doi:10.1136/bmjopen-2018-024162

Watson, W. A., Litovitz, T. L., Klein-Schwartz, W., Rodgers, G. C., Youniss, J., Reid, N., \& Borys, D. (2004). 2003 Annual Report of theAmerican Association of Poison Control Centers Toxic Exposure Surveillance System. The American Journal of Emergency Medicine, 22(5), 335-404. doi: 10.1016/j.ajem.2004.06.001

Werner, P. D. (1977). Implications of AttitudeBehavior Studies for Population Research and Action. Studies in Family Planning, 8(11), 294. doi:10.2307/1966280

Wilkinson, A., Parker, M., Martineau, F., \& Leach, M. (2017). Engaging 'Communities': Anthropological insights from the West African Ebola Epidemic. Philosophical Transactions of the Royal Society B: Biological Sciences, 372(1721), 20160305. doi:10.1098/rstb.2016.0305

World Health Organization (WHO). (2005). World Health Statistics (pp. 1-59, Rep. No. Part 1). 20 Avenue Appia, Switzerland: WHO Press.

World Health Organization (WHO). (2010). Guidelines for poison control. Retrieved January 13, 2019, from https://www.who. int/ipcs/publications/training_poisons/ guidelines_poison_control/en/index $5 . \mathrm{html}$

World Health Organization (WHO). (2012). Poisoning Prevention and Management. Retrieved November 4, 2018, from https:// www.who.int/ipcs/poisons/en/

World Health Organization (WHO). (2019). Suicide. Retrieved November 18, 2019, from https://www.who.int/news-room/factsheets/detail/suicide. 